\title{
Corrigendum
}

\section{Which is the most preventive measure against tail damage in finisher pigs: tail docking, straw provision or lowered stocking density? - CORRIGENDUM}

\author{
M. L. V. Larsen, H. M.-L. Andersen and L. J. Pedersen \\ doi:10.1017/S175173111700249X, published by Cambridge University Press, 2 November 2017.
}

The original publication of Model 1 within the Results Section contained an error within the last sentence. The sentence should have read as follows;

However, the effect of tail docking tended to be higher than the effect of straw provision $(P=0.092)$.

The authors apologise for the error.

\section{Reference}

Larsen MLV, Andersen H M.-L and Pedersen LJ 2017. Which is the most preventive measure against tail damage in finisher pigs: tail docking, straw provision or lowered stocking density? Animal, first published online 2 November 2017, doi: 10.1017/S175173111700249X. 